\title{
Valoración del Aprendizaje Basado en Problemas por los alumnos: diferencias por sexo
}

\author{
Mercedes CARMOna MarTíneZ \\ Universidad Católica San Antonio de Murcia \\ mcarmona@ucam.edu \\ Ma Carmen Conesa Pérez \\ Universidad Católica San Antonio de Murcia \\ ccperez@ucam.edu \\ $M^{\mathrm{a}}$ Isabel Ros Clemente \\ Universidad Católica San Antonio de Murcia \\ irclemente@ucam.edu
}

\begin{abstract}
Resumen:
Esta investigación pretende valorar la aplicación de la metodología del Aprendizaje Basado en Problemas (ABP) atendiendo al género de los alumnos. En numerosas investigaciones se observan diferencias en las estrategias y estilos de aprendizaje en cuanto al sexo que pueden influir en la valoración de un método docente concreto. Tras la aplicación de una encuesta donde se evalúa la eficacia del procedimiento de aprendizaje aplicado, se obtiene que si bien realizado el contraste de igualdad de medias en la mayoría de los casos, las diferencias no han sido significativas en cuanto al género de los alumnos en dicha valoración.
\end{abstract}

Palabras clave: Aprendizaje basado en problemas; metodología docente; diferencias por sexo.

\section{Student Assessment of Problem-based Learning: differences by gender}

\begin{abstract}
:
This research aims to evaluate the application of Problem Based Learning (PBL) methodology in response to students' gender. Numerous investigations observe differences in learning styles and strategies as for gender that can influence the assessment of a particular teaching method. After conducting a survey which evaluates the effectiveness of applied learning procedures, we find that despite contrast was made by equal average in most cases, the differences were not significant in terms of student gender in such assessment.
\end{abstract}

Key Words: Problem-based learning; teaching methodology; gender differences.

\section{Referencia normalizada:}

Carmona Martínez, M., Conesa Pérez, M.C y Ros Clemente, M.I. (2014): Valoración del aprendizaje basado en problemas por los alumnos: diferencias por sexos. Historia y Comunicación Social. Vol. 19. Núm. Especial Enero. Págs. 725-734.

Sumario: 1. Introducción. 3. Metodología empleada. 4. Análisis de los resultados de la investigación. 5. Conclusiones. 6. Referencias bibliográficas. 


\section{Introducción}

Los alumnos tienden a adoptar un enfoque de aprendizaje que depende de la motivación para aprender y de la percepción de la situación de aprendizaje (Marton y Säljö, 1984). Para Kolb (1984) el estilo de aprendizaje es la respuesta del estudiante a los estímulos y a la utilización de éstos en el contexto de aprendizaje.

Las nuevas estrategias metodológicas incorporadas en el ámbito de Espacio Europeo de Educación Superior (EEES) serán afrontadas por alumnos y alumnas según un estilo de aprendizaje propio. Las diferencias de género en este proceso, dependerán del contexto y del tipo de carrera estudiada. Cano (2000). En la investigación llevada a cabo por dicho autor, en estrategias de aprendizaje las alumnas superan a los alumnos en motivación, interés y actitud para el estudio, administración del tiempo, ayudas de estudio, repaso y comprobación de los niveles de comprensión logrados, detectando un mayor nivel de ansiedad y miedo al fracaso. En cuanto a estilos de aprendizaje, dicho estudio, obtiene mayor puntuación por parte de los alumnos en el estilo de procesamiento profundo de la información y mejor puntuación por parte de las alumnas en el estilo de estudio metodológico, no existiendo diferencias de procesamiento profundo en carreras de ciencias, ni de estudio metódico en letras.

En la investigación llevada a cabo por Martín y Camarero (2001) se confirma que alumnos y alumnas utilizan estilos y estrategias diferenciales que optimizan su rendimiento, reflejando un menor empleo de técnicas de estrategias de aprendizaje en los alumnos frente a las alumnas, y denotando por parte de éstos un estudio menos metódico al margen de la especialidad cursada.

Las diferentes formas de abordar y procesar la información por parte de los alumnos se verán afectadas por el tipo de contenidos curriculares y las exigencias de la tarea universitaria (Martín y Camarero, 2001). El profesor universitario deberá elegir entre las distintas técnicas de aprendizaje dependiendo del tipo de competencias a desarrollar, las características del grupo y las posibilidades que nos ofrece el escenario donde vamos a realizar la actividad (De Miguel, 2006).

Para el desarrollo de competencias en una titulación de empresa y más concretamente en el área de análisis contable se han de aplicar técnicas o herramientas que apliquen los conocimientos a la práctica. Dentro de las técnicas que permiten un aprendizaje significativo o por descubrimiento se ubica el Aprendizaje Basado en Problemas (ABP) que tiene por finalidad desarrollar aprendizajes activos a través de la resolución de problemas (De Miguel, 2005).

En método ABP se parte de la idea de que el estudiante aprende de un modo más adecuado cuando tiene la posibilidad de experimentar, ensayar o indagar sobre actividades cotidianas (De Miguel, 2005), es una metodología de aprendizaje que consiste en construir el conocimiento sobre la base de problemas de la vida real (Font, 2004) siendo el problema el foco organizador que dirige el proceso para el desarrollo de habilidades y competencias simulando situaciones a las que luego se enfrentaran los 
alumnos en la práctica profesional (Morales y Landa, 2004, Font, 2004, Restrepo, 2005).

El objetivo de este trabajo será la valoración de la aplicación de la técnica de ABP por parte de los alumnos observando las diferencias de valoración atendiendo al género de los mismos.

\section{Metodología empleada}

Con la finalidad de que las competencias sean adquiridas se ha planteado la metodología del aprendizaje basado en el ABP. Los alumnos se han distribuido en grupos de trabajo de un máximo de tres personas, donde siguiendo el método publicado en Journal of $P B L^{I}(2000)$ se les reta a estudiar la viabilidad económica de una empresa de nueva creación. Establecido este problema abierto y semiestructurado se fijaron ocho tareas a seguir. En primer lugar, indagaron en el problema y exploraron la situación patrimonial y financiera inicial de su empresa. En segundo lugar, investigaron en la solución del problema con los conocimientos previos de que disponían. En tercer lugar, identificaron los conocimientos que debían adquirir. En la cuarta tarea, jerarquizaron sus necesidades de nuevos aprendizajes, fijaron su objetivo y los recursos de información a utilizar, distribuyéndose las tareas entre los participantes. La quinta tarea consistió en el autoestudio y preparación. La sexta, fue la puesta en común de la información. En séptimo lugar, se utilizaron los conocimientos previos y adquiridos en la redacción de un informe sobre la viabilidad económica de su empresa. Por último, se evaluó el aprendizaje logrado, la solución dada y la efectividad de todo el proceso, mediante un cuestionario.

Este trabajo quiere, una vez analizado el grado de satisfacción de los alumnos por el uso de esta metodología, comprobar si existen diferencias por sexos a la hora de percibir la eficacia del método de aprendizaje utilizado.

La evaluación de la metodología de aprendizaje se ha llevado a cabo mediante una encuesta a los 83 alumnos de la asignatura Análisis de Estados Contables del segundo curso en $\mathrm{ADE}$. La encuesta ${ }^{2}$ utilizada está dividida en cuatro bloques para facilitar la obtención de información según las distintas áreas que se pretenden analizar. El primer bloque recoge información personal del alumno, sexo y edad. En los restantes bloques el alumno debe valorar entre 1 (muy en desacuerdo) y 5 (muy de acuerdo), diversos aspectos del aprendizaje mediante el $\mathrm{ABP}$, el desarrollo de varias competencias generales y específicas, así como una serie de características del método.

Para el tratamiento de los datos conseguidos utilizaremos métodos del análisis descriptivo con la finalidad de extraer conclusiones sobre la efectividad de la metodología de aprendizaje desarrollada. Posteriormente, se analizarán si existen diferencias por sexo desarrollando contrastes de igualdad de medias. Para realizar estos contrastes se ha comprobado, en primer lugar que las variables analizadas verifican el cumplimiento de los supuestos de normalidad y homocedasticidad (igualdad de 
varianzas). En los casos en que las variables cumplen ambos supuestos, se ha aplicado el test $t$ de igualdad de medias.

Con respecto al supuesto de normalidad, la mayoría de variables lo cumplen. Como vemos en la tabla 1, tan solo CE8 y CE13 no siguen la distribución gaussiana, según el test de Kolmogorov-Smirnov (a un 95\%):

Tabla 1. Test de Normalidad de Kolmogorov-Smirnov

\begin{tabular}{|l|l|l|}
\cline { 2 - 3 } \multicolumn{1}{c|}{} & Z de K-S & Sig. \\
\hline CE8 & 1,366 &, 048 \\
\hline CE13 & 1,366 &, 048 \\
\hline
\end{tabular}

En estos casos, no se ha aplicado el test $\mathrm{t}$ de igualdad de medias sino la prueba no paramétrica de Mann-Whitney.

Con respecto al supuesto de homocedasticidad, la mayoría de variables lo cumplen. Tan solo P19, CG1 y CE13 no presentan varianzas iguales en ambas submuestras, según el test de Levene (ver tabla 2):

Tabla 2. Test de Homocedasticidad de Levene

\begin{tabular}{|l|l|c|}
\cline { 2 - 3 } \multicolumn{1}{c|}{} & $\begin{array}{l}\text { Estadístico } \\
\text { de Levene }\end{array}$ & Sig. \\
\hline P19 & 5,337 &, 023 \\
\hline CG1 & 4,774 &, 032 \\
\hline CE13 & 4,969 &, 029 \\
\hline
\end{tabular}

En los dos primeros casos se ha aplicado se ha aplicado el test $t$ de igualdad de medias con la correspondiente corrección para varianzas que no son iguales ${ }^{3}$. En el tercer caso no fue necesario porque se realizó la prueba de Mann-Whitney.

\section{Análisis de los resultados de la investigación}

En nuestro estudio la muestra está compuesta por 83 alumnos, de los cuales el $59 \%$ son hombres con una media de edad de 25,22 años y el $41 \%$ mujeres de edad media 26,32 años.

La puntuación obtenida de media por los distintos métodos de enseñanza analizados (lección magistral, ABP, estudio de casos y manuales) supera en todos los casos los cinco puntos, siendo el estudio de casos el que obtiene una mayor puntuación (6,98 puntos sobre 10) y alcanzando el mínimo los libros de texto con una puntuación global de 5,15 puntos. 
Gráfico 1. Valoración media de los distintos métodos de aprendizaje

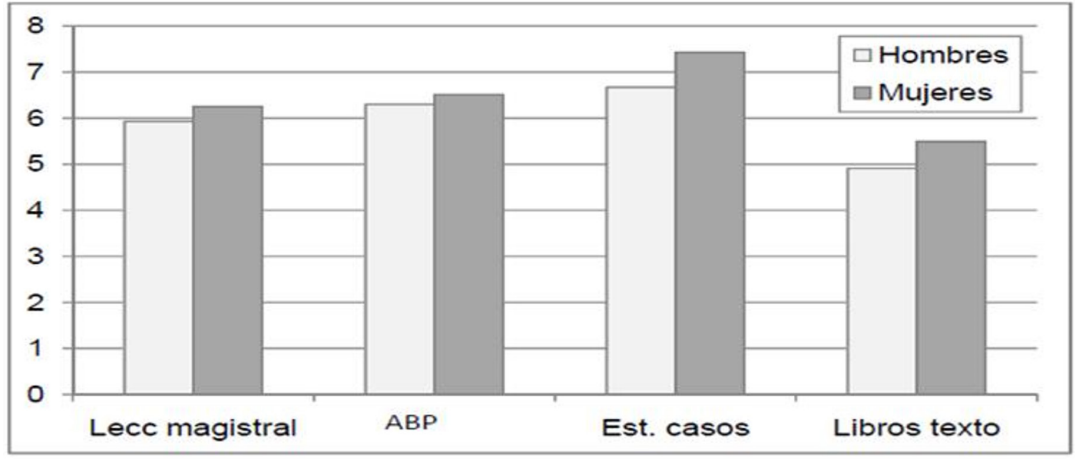

Si diferenciamos las puntuaciones por sexos (gráfico 1) observamos que en todos los casos la valoración dada por las mujeres supera a la valoración hecha por los hombres. Con el objetivo de analizar si esa diferencia en las medias es significativa se ha realizado la prueba $\mathrm{T}$ para la igualdad de medias comprobándose que en todos los casos la sig. bilateral es superior a 0,025 , no pudiéndose rechazar la hipótesis nula (las medias son iguales para hombres y mujeres). Por lo tanto, con un nivel de confianza del $95 \%$, no se detectan diferencias estadísticamente significativas en la puntuación que dan ellos y ellas a cada uno de los métodos de enseñanza.

En la evaluación de los distintos elementos del método de aprendizaje basado en ABP apreciamos que los resultados son muy positivos, la valoración media de los aspectos oscila entre el 3,68 (en P10) y el 4,30 (en P25). Al indagar si las diferencias son significativas por sexos obtenemos que solo en el aspecto P28 "En el ABP los conocimientos del grupo son importantes para realizar el plan de viabilidad de una empresa" se detecta una diferencia estadísticamente significativa, con un nivel de confianza del $95 \%$, entre la valoración de hombres y mujeres, pues, para este ítem, Sig. es menor a 0,025 (se rechaza la hipótesis nula de igualdad de medias $\mu_{\mathrm{H}}=\mu_{\mathrm{M}}$ ).

En ese caso, si se analizan los estadísticos descriptivos de P28 para cada uno de los grupos se puede comprobar que la valoración media de las mujeres $(4,44$ puntos) es superior a la de los hombres $(4,00)$.

Según los resultados ofrecidos por el estadístico t bajo la hipótesis nula de igualdad de medias, el grado de acuerdo con la adquisición de las competencias generales entre ambos sexos, para un nivel de confianza del 95\%, no es estadísticamente diferente entre hombres y mujeres en 16 de los 17 casos considerados. Tan solo en la competencia CG10 "Participar en el ABP es una experiencia creativa" se detecta una diferencia estadísticamente significativa entre la valoración de hombres y mujeres, pues, para este ítem se rechaza la hipótesis nula de igualdad de medias.

Analizando los estadísticos descriptivos de CG10 para cada uno de los grupos se puede comprobar que la valoración media de las mujeres $(4,41)$ es superior a la de los hombres $(4,06)$. 
Al estudiar cómo perciben la adquisición de competencias específicas a través del ABP, sí se aprecian numerosas diferencias estadísticamente significativas entre hombres y mujeres (casos en los que Sig. es menor a 0,025 y, por lo tanto, se rechaza la hipótesis nula de igualdad de medias): CE1, CE3, CE8, CE12 y CE13.

Si se analizan los estadísticos descriptivos de dichas variables para cada uno de los grupos se puede comprobar que la valoración media de las mujeres es en todos y cada uno de los casos superior a la de los hombres. En el gráfico 2 se presentan las puntuaciones medias dadas por cada sexo sobre su percepción de cómo la metodología de aprendizaje ayuda a la adquisición de una serie de competencias específicas.

Gráfico 2. Valoración de la adquisición de competencias específicas

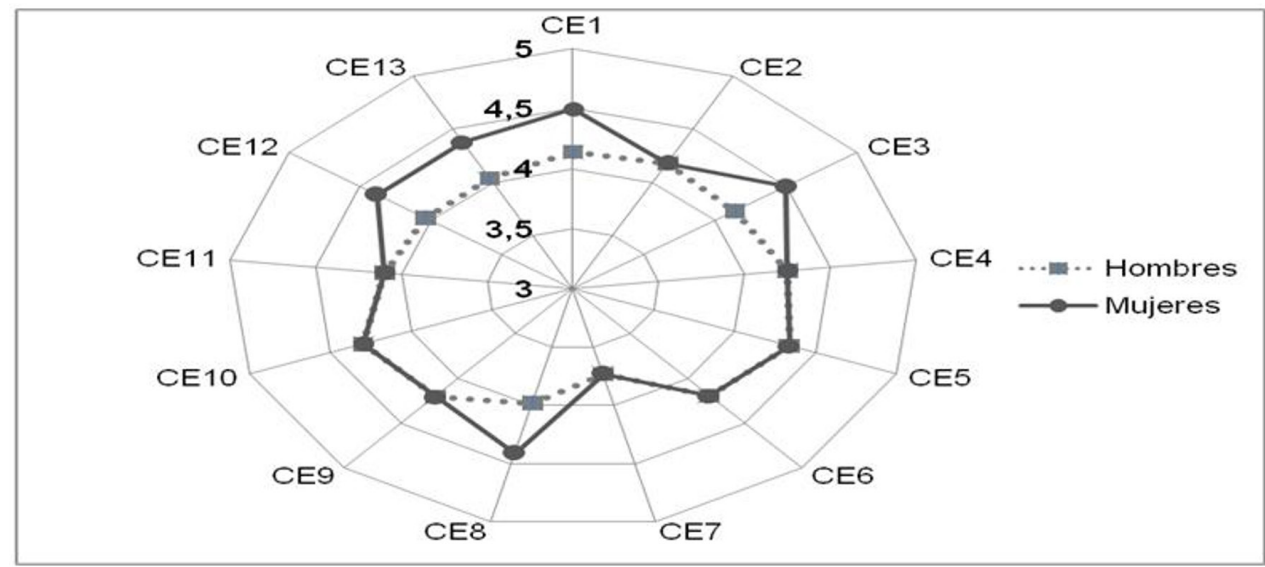

Por último, al contrastar si el grado medio de acuerdo de hombres y mujeres acerca de las características generales del ABP es estadísticamente diferente o no, no se detectan diferencias estadísticamente significativas en el grado medio de acuerdo de hombres y mujeres acerca de las características generales del ABP.

\section{Conclusiones}

Nuestros resultados indican que la valoración que los alumnos realizan sobre la eficacia de la metodología docente ABP es positiva, como se ha podido comprobar por la alta puntuación obtenida tanto en los aspectos generales del método como en la adquisición de competencias.

Al analizar los resultados clasificados por sexos, se observa que el grado de acuerdo entre los dos sexos es muy elevado presentándose diferencias significativas solamente en algunos casos. 
Tanto en la puntuación de los distintos métodos de enseñanza alternativos como en las características del método aplicado no se ha podido rechazar la hipótesis nula de igualdad de medias para ninguno de los ítems. Es decir, existe un elevado grado de acuerdo entre hombres y mujeres.

En los diversos aspectos del aprendizaje mediante ABP solamente en uno de ellos encontramos diferencias estadísticamente significativas en el aspecto P28 en el que se valoran los conocimientos del grupo para resolver el problema planteado. Dicha diferencia puede obedecer a que, como se ha comprobado en estudios previos, las alumnas están más abiertas a recibir ayudas en el estudio.

La conclusión extraída del análisis estadístico para las competencias generales destaca que participar en el método de aprendizaje ABP es una experiencia creativa, valorada por las alumnas en mayor grado que por los alumnos, lo que demuestra mayor propensión a la innovación docente por parte de ellas. Esta afirmación se ha comprobado mediante la prueba t con un nivel de confianza del $95 \%$.

Por último, donde mayores diferencias estadísticamente significativas encontramos entre la valoración hecha por hombres y mujeres es en la percepción de la adquisición de competencias específicas. En concreto, las competencias, comprensión del plan de viabilidad, resolver el caso adaptado a la realidad, saber interpretar la situación económico-financiera, identificar y analizar fuentes de información contable y financiera, coinciden en contenido con el objetivo perseguido de adquirir conocimientos a través del planteamiento de un problema.

La mayor valoración de media hecha por las alumnas puede responder a que éstas superan a los alumnos en motivación, interés y actitud para el estudio, administración del tiempo, ayudas de estudio, repaso y comprobación de los niveles de comprensión logrados, detectando un mayor nivel de ansiedad y miedo al fracaso como señala en su estudio Cano (2000).

\section{Bibliografía}

CANO, F. (2000). "Diferencias de género en los procesos de aprendizaje en universitarios". Psicotherma, vol.12, no3, p. 360-367.

DE MIGUEL, M. (2005). Modalidades de enseñanza centradas en el desarrollo de competencias. Oviedo: Ediciones Universidad de Oviedo.

DE MIGUEL, M. (2006). "Metodologías para optimizar el aprendizaje. Segundo objetivo del Espacio Europeo de Educación Superior". Revista Interuniversitaria de Formación del Profesorado, $\mathrm{n}^{\circ} 20$ vol. 3, p. 71-91

FONT, A. (2004). "Líneas maestras del Aprendizaje por Problemas". Revista Interuniversitaria de Formación del Profesorado, no 18 vol. 1, p. 79-95.

KOLB, D.A. (1984). Experiential learning. Experience as the source of learning and development. Englewood Cliffs, N.J.: Prentice Hall, Inc. 
MARTÍN, F. y CAMARERO, F. (2001). "Diferencias de género en estrategias y estilos de aprendizaje". Psicotherma, vol. 13, nº 4, p. 598-604.

MARTON, F. y SÄLJÖ, R. (1984). Approaches to learning. En F. Marton, D.J. Hounsell, y N.J. Entwistle (Eds.). The experience of learning. Edinburgh: Scottish Academic Press.

MORALES, P. Y LANDA, V. (2004). “Aprendizaje Basado en Problemas. ProblemBased Learning". Theoria, vol. 13, p. 145-157.

RESTREPO, B. (2005). "Aprendizaje basado en problemas (ABP)". Educación y Educadores, vol. 8, p. 9-19.

\section{Anexo}

Aspectos del aprendizaje valorados: P 5. El ABP ha contribuido a la adquisición de nuevos conocimientos -P 6. El ABP me ha permitido aplicar los conocimientos teóricos a un entorno realista -P 7. El ABP puede ser útil como complemento de otros métodos de enseñanza alternativos (estudio de casos, lección magistral y libros de texto) -P 9. El ABP ayuda a comprender la información de la empresa e interpretarla adecuadamente -P 10. El ABP ayuda a interrelacionar conceptos de otras asignaturas -P 11. El ABP ayuda a afianzar conocimientos -P 12. El ABP ayuda a formar una idea aproximada de la importancia de llevad a cabo el plan de viabilidad de una empresa -P 13. El ABP ayuda a crear una idea y ver si es viable -P 14. El ABP ayuda a proponer e implementar soluciones eficaces a los problemas encontrados -P 15. El ABP ayuda a reconocer la necesidad de cambios -P 16. El ABP ayuda a avaluar el impacto probable de las decisiones -P 17. El ABP ayuda al desarrollo de habilidades creativas -P 18. El ABP ayuda al desarrollo de habilidades para el trabajo en equipo -P 19. El ABP ayuda al desarrollo de habilidades emprendedoras -P 20. El ABP ayuda al desarrollo de la interdisciplinariedad de las materias que forman el plan de estudios -P 21. La simplificación de las decisiones en un ABP NO es excesiva y refleja la realidad -P 22. El ABP despierta la colaboración entre los jugadores -P 23. Participar en un $\mathrm{ABP}$ es una experiencia creativa -P 24. El ABP permite que el aprendizaje se base en la creatividad propia del alumno -P 25. El ABP permite conocer y analizar si una empresa o plan de empresa es viable económicamente -P 26. En el ABP las decisiones no se toman de forma aislada se tienen en cuenta resultados anteriores -P 27. En el ABP, la cohesión del grupo es importante para llevara a cabo el plan de viabilidad de una empresa -P 28. En el ABP, los conocimientos del grupo son importantes para realizar el plan de viabilidad de una empresa -P 29. En el ABP, mis conocimientos son importantes para realizar el plan de viabilidad de una empresa -P 30. En el ABP, el esfuerzo personal es necesario para realizar el plan de viabilidad de una empresa -P 31. En el ABP, la cohesión del grupo es importante para conseguir un adecuado aprendizaje -P 32. En el ABP, los conocimientos del grupo son importantes para conseguir un adecuado aprendizaje. -P 33. En el ABP, mis conocimientos son importantes para conseguir un adecuado aprendizaje -P 34. En el ABP, el esfuerzo personal es necesario para conseguir un adecuado aprendizaje -P 35. En el ABP 
El esfuerzo requerido para el desarrollo del juego está acorde con los resultados de aprendizaje obtenidos.

Competencias generales: CG 1. Aplicar conceptos teóricos a la práctica -CG 2. Entender la realidad empresarial - CG 3. Analizar y gestionar la información -CG 4. Resolver problemas -CG 5. Tomar decisiones -CG 6. Gestionar el tiempo -CG 7.Manejo de nuevas tecnologías -CG 8. Aprendizaje -CG 9. Comunicación-CG 10. Reflexión -CG 11.Trabajo individual -CG 12.Trabajo en equipo -CG 13.Organización y planificación -CG 14.Capacidad de innovación -CG 15.Espíritu emprendedor -CG 16.Creatividad -CG 17.Aprendizaje autónomo

Competencias Específicas: CE 1. Comprensión del Plan de viabilidad para una empresa -CE 2. Analizar la información contenida en los EE.FF -CE 3. Saber interpretar la situación econ-fin que transmiten -CE 4. Resolver el caso planteado adaptado a la realidad en equipo -CE 5. Elaborar el Balance y Cuenta de Pérdidas y Ganancias -CE 6. Desarrollar información contable necesaria para la toma de decisiones -CE 7. Integrar la ética en las decisiones -CE 8. Resolver el caso planteado adaptado a la realidad en equipo -CE 9. Elaborar proyectos de viabilidad económico-financiera -CE 10. Relacionar la información obtenida, con la evolución econ-financiera -CE 11. Interpretar los resultados obtenidos -CE 12. Identificar fuentes de información contable y financiera -CE 13. Analizar información financiera -CE 14. Utilizar terminología contable y financiera correcta

Características: $\mathrm{CH}$ 1. Participación activa del alumno -CH 2. Diversión -CH 3. Complementariedad con otros métodos docentes - $\mathrm{CH} 4$. Tiempo empleado - $\mathrm{CH} 5$. Motivación del alumno-CH 6. Complejidad -CH 7. Cercanía a la realidad

\section{Notas}

1. ABP

2. Cuestionario elaborado a partir del desarrollado en Carmona, 2008.

3. El estadístico de contraste se calcula a partir de una varianza promediada entre las varianzas de cada grupo.

\section{Las autoras}

María Mercedes Carmona Martínez es profesora de Econometría y de Estadística en la Universidad Católica San Antonio de Murcia (UCAM), subdirectora de la Titulación de Administración y Dirección de Empresas en la UCAM, coordinadora 
de "Research Methodology Module" del English PhD Programme en la UCAM, y coordinadora del título International Business Certificate de la UCAM y la Hochschule für Oekonomie \& Management (FOM) de Alemania. Licenciada en Economía y Doctora en Economía por la Universidad de Murcia. Especialidad en análisis de datos, trabajando en las líneas de investigación de Adopción y Difusión de Innovaciones e Innovación Educativa en Economía y Empresa. Ha presentado numerosas comunicaciones a congresos nacionales e internacionales (CUICIID, INTED, EDULEARN,...), y ha publicado artículos de investigación en varias revistas de impacto JCR/IN-RECS (Estudios sobre Educación, Revista de Economía Aplicada, Revista de Estudios Regionales). Ha participado en la elaboración de manuales docentes universitarios. Ha codirigido y está codirigiendo diversas tesis doctorales del ámbito de las ciencias sociales, que hacen uso de una metodología cuantitativa aplicada a diversos fenómenos.

María del Carmen Conesa Pérez es profesora de Contabilidad Financiera en la Universidad Católica San Antonio de Murcia. Licenciada en Administración y Dirección de Empresas. Tesis de Master en el programa de doctorado en Ciencias de La Empresa de la Universidad de Murcia. Proyecto de tesis doctoral: Determinantes de la Eficiencia en la Prestación de los Servicios Públicos Municipales. Miembro del Grupo de Investigación "Organización de empresas y marketing". Responsable de la materia Contabilidad y Finanzas del MBA (UCAM). Publicaciones en revista JCR "Estudios sobre el Mensaje Periodístico"

María Isabel Ros Clemente es profesora de Contabilidad y Análisis de los estados contables en la Universidad Católica San Antonio de Murcia. Licenciada en Ciencias Económicas y Empresariales. Suficiencia Investigadora en el programa de Administración y Dirección de Empresas: Organización y Marketing. Proyecto de tesis doctoral: Inserción laboral de personas discapacitadas. Miembro del Grupo de Investigación "Organización de empresas y marketing". Responsable de la materia Contabilidad y Finanzas del MBA (UCAM). Publicaciones en revista JCR "Estudios sobre el Mensaje Periodístico" 